\title{
MUPPscore: An R Script for Expected A Posteriori Scoring of Multi-Unidimensional Pairwise Preference Items
}

\author{
Li (Ada) Guan ${ }^{1,2}$, Tianjun Sun ${ }^{3,4}$, and Nathan T. Carter ${ }^{2}$ \\ ${ }^{1}$ People Analytics, Aon \\ ${ }^{2}$ Department of Psychology, University of Georgia \\ ${ }^{3}$ Department of Psychology, University of Illinois at Urbana-Champaign \\ ${ }^{4}$ Department of Psychological Sciences, Kansas State University
}

DRAFT version.

Please do not copy or cite without the authors' permission.

\section{Author Note:}

Ada Guan is now a senior data scientist at Aon. This manual originates from her work during doctoral training at the University of Georgia.

Tianjun Sun is currently a $\mathrm{PhD}$ candidate in psychology at the University of Illinois and will be an assistant professor of psychological sciences at the Kansas State University starting in August 2021.

Nathan T. Carter is an associate professor and the program chair of industrial-organizational psychology at the University of Georgia.

Correspondence regarding this manual should be addressed to Tianjun Sun at tsun5@illinois.edu. 


\begin{abstract}
In this manual, we present a flexible and freely available tool for obtaining latent trait scores from multi-unidimensional pairwise preference (MUPP) tests: An R script named MUPPscore. The development of the MUPPscore script provides a solution to the issue that is the previously inconvenient estimation of forced choice item pairs. Instead of using the computationally-intensive multidimensional Bayes modal procedure, the MUPPscore script employs the expected a posterior (EAP) scoring procedure, which provides plausible latent trait score estimates and is also consistent with scoring algorithms used in existing software programs intended for single stimulus measures (e.g., GGUM2004, IRTPRO). The MUPPscore script also returns the empirical marginal reliability of EAP theta estimates and outputs a series of files that can be used to easily create and modify three-dimensional surface charts for plotting MUPP item response function (IRF) in Microsoft Excel.
\end{abstract}

\title{
Keywords:
}

item response theory; multi-unidimensional pairwise preference; forced-choice measures; Bayesian estimation; R program 


\section{MUPPscore: An R Script for Expected A Posteriori Scoring of Multi-Unidimensional Pairwise Preference Items}

Recent research has presented evidence that personality assessment conducted using forced-choice (FC) formats may recover more accurate estimates of personality traits than traditional self-report methods. In particular, researchers have been interested in this approach to personality testing for high-stakes situations in which respondents are motivated to distort their responses to appear more favorable, such as employment testing (e.g., Chernyshenko et al., 2009; Drasgow et al., 2012). Under this approach to personality testing, respondents are asked to choose between two statements, each designed to tap into a different personality trait, which is presumed to lower the chances that respondents can successfully distort responses. However, as Chernyshenko et al. (2009) have indicated, multi-statement multidimensional FC items may increase the cognitive load on respondents leading to greater errors, a less accurate score may occur due to the complex judgement process. Further, recent results have questioned the ability of multidimensional FC tests to solve the problem of response distortion in employment tests (Guan et al., 2015). Given this recent surge of interest and its application to crucial and highprofile testing programs, such as the United States Army's tailored adaptive personality assessment system (TAPAS; see Drasgow et al., 2012), more research on this method of assessment is greatly needed.

For research in this area to progress, a flexible and freely available tool for obtaining latent trait scores from multi-unidimensional pairwise preference (MUPP) tests should be essential in both research and applied settings. However, no such program is currently publicly available to conveniently accomplish latent trait score estimation for the MUPP model. The development of the MUPPscore script provides a solution to this issue. Instead of using the 
computationally-intensive multidimensional Bayes modal procedure, the MUPPscore script employs the expected a posterior (EAP) scoring procedure, which provides plausible latent trait score estimates (see Hontangas et al., 2015) and is also consistent with scoring algorithms used in software programs intended for single stimulus measures (e.g., GGUM2004, IRTPRO). Note that our EAP algorithm does not integrate across dimensions (unlike the Bayes modal procedure adopted by Stark et al. [2005] which would greatly complicate and bog down the scoring) and should be considered a more simplistic approach. The MUPPscore script also returns the empirical marginal reliability of EAP theta $(\theta)$ estimates and outputs a series of files that can be used to easily create and modify three-dimensional surface charts for plotting MUPP item response function (IRF) in Microsoft Excel.

\section{Technical Details of the MUPPscore Script}

The MUPPscore script utilizes the generalized graded unfolding model (GGUM; Roberts et al., 2000) item parameters as the basis for the MUPP model. Although the MUPP model can be implemented with any item response theory (IRT) model as its basis, the GGUM has been used in all available research and applications of the MUPP model. The full GGUM for dichotomous data, which is adapted in the MUPPscore script is stated as:

$$
P\left(X_{i}=1 \mid \theta_{j}\right)=\frac{\exp \left[\alpha_{i}\left(\theta_{j}-\delta_{i}-\tau_{i}\right)\right]+\exp \left[\alpha_{i}\left[2\left(\theta_{j}-\delta_{i}-\tau_{i}\right)\right]\right]}{1+\exp \left[\alpha_{i}\left[3\left(\theta_{j}-\delta_{i}\right)\right]\right]+\exp \left[\alpha_{i}\left(\theta_{j}-\delta_{i}-\tau_{i}\right)\right]+\exp \left[\alpha_{i}\left[2\left(\theta_{j}-\delta_{i}-\tau_{i}\right)\right]\right]} .
$$

The GGUM states that the probability that the response to the item, $P\left(X_{i}=1 \mid \theta_{j}\right)$, will be affirmative as a function of (i) the latent trait standing of person $j$ (denoted as $\theta_{j}$ ), (ii) the location of the of the $i^{\text {th }}$ item on the latent trait continuum (denoted as $\delta_{i}$ ), (iii) the discrimination of the item (denoted as $\alpha_{i}$ ), and (iv) the location of the item threshold (denoted as $\tau_{i}$ ).

In applying the MUPP model, the focal trait (i.e., the trait to be estimated, $\theta_{\mathrm{s}}$ ) is estimated by first estimating the unidimensional GGUM in Equation (1) utilizing data in which persons 
selecting the statement reflecting $\theta_{\mathrm{s}}$ receive a score of $X_{\mathrm{s}}=1$, whereas those choosing the statement reflecting a trait other than $s$ (i.e., the non-focal trait, denoted as $t$ ) receive a score of $X_{s}$ $=0$. Next, a new dataset is created in which all responses are reverse-scored, such that those whom choose option $s$ receive a score of $X_{t}=0$, and those choosing option $t$ receive a score of $X_{t}$ $=1$. The GGUM is then estimated with these data. Using the two resulting sets of estimated item parameters as a starting point, the MUPP can be stated as:

$$
P\left(s>t \mid \theta_{s}, \theta_{t}\right)=\frac{P\left(X_{s}=1 \mid \theta_{s}\right) P\left(X_{t}=0 \mid \theta_{t}\right)}{P\left(X_{s}=1 \mid \theta_{s}\right) P\left(X_{t}=0 \mid \theta_{t}\right)+P\left(X_{s}=0 \mid \theta_{s}\right) P\left(X_{t}=1 \mid \theta_{t}\right)}
$$

The MUPP states that the probability that the person will choose option $s$ over option $t$ is a function of the conditional probabilities derived from the item parameters of the two aforementioned datasets under the unidimensional GGUM. The MUPPscore script calculates these probabilities and uses them as inputs for the calculation of EAP $\theta$ estimates. This is accomplished by evaluating the likelihood of each response pattern at various potential levels of $\theta$ (i.e., quadrature points) and calculating the expected value and standard error of $\theta$ from these likelihoods by mixing them with theoretical likelihoods based on the assumption that $\theta$ is distributed normally with a mean of 0 and unit variance.

\section{Demonstration of the MUPPscore Script}

To demonstrate the capability of the MUPPscore script, we first compared our scoring using the simplistic EAP approach to the latent trait scores estimated using Stark et al.' (2005) Bayes modal approach. Using an empirical dataset, designed to measure the Five Factor Model of personality, 1,130 participants responded to 46 MUPP pairs to reflect their personality dimensions: Agreeableness (8 pairs), Extraversion (10 pairs), Conscientiousness (10 pairs), Openness (8 pairs) and Emotional Stability (10 pairs). Latent trait score estimations were consistent with the Bayes modal approach (Boyce et al., 2015) as same-trait correlations 
were $.81, .94, .93, .90$ and .80 , respectively. With regard to the processing speed, each run of the MUPPscore script required about 20 seconds to complete.

To demonstrate the accuracy of estimates calculated using MUPPscore, we simulated a 20-pair, two-dimensional MUPP test. Latent trait scores (i.e., $\theta$ s) for two traits were simulated from a multivariate normal distribution with $\mu=0$ and $\sigma=1$ for both traits; the correlation between two traits was set to .20. Random discrimination values (i.e., $\alpha_{i}$ ) were sampled from a uniform distribution with values ranging from 1 to 2 to represent both moderate and high discrimination. Item location parameters (i.e., $\left.\delta_{i}\right)$ were generated from a uniform $(-2,2)$ distribution, and response thresholds (i.e., $\tau_{i}$ ) were simulated from a uniform distribution with values ranging from -2 to -1 . Responses were simulated for a sample size of 2,000 . Using the simulated dataset, the correlation between the true theta and estimated theta for the 20-pair test was .72, consistent with the results of Hontangas et al. (2015) under similar data-analytic conditions. Overall, this program has the capability of delivering plausible latent trait estimates for the MUPP model.

\section{Use of the MUPPscore Script}

MUPPscore is a user-defined function script written in the free software programming language R (R Core Team, 2018). Source code for the MUPPscore can be found in Appendix A. The MUPPscore script is free-of-charge for noncommercial use only. The MUPPscore script estimates one trait (i.e., focal trait) for each run with no limit on the number of respondents or the number of total pairs. There are a few precursory steps before the MUPPscore can execute successfully. First, the user needs to specify the working directory to indicate where the input files are. Notably, all \in the file location must be replaced with a / or $\backslash$. The working directory can be replaced in the first line of the $\mathrm{R}$ code. Second, the users also need to install and load the 
LearnBayes R package (Albert, 2018). In addition, the user needs to specify the number of respondents (i.e., N), and the number of total pairs (i.e., PAIRS) in the following two lines of the $\mathrm{R}$ code to reflect the dataset. Next, the user needs to place two files that are in .csv format under the same folder specified in the working directory: 1) a "Parameters.csv" file of two statements for each pair (i.e., $\alpha, \delta$, and $\tau$ ), and the format can be found in Figure 1; and 2) a "Responses.csv" file of the focal trait only (i.e., 0 for non-endorsement, 1 for endorsement, and code 9 for missing values), it is important to note that the order of responses must be consistent with the pair order specified in the item parameters file. Then, the user can now run the program after all these steps are properly completed.

The MUPPscore will produce three sets of output files and will be stored under the same folder specified in the working directory: 1) a "TraitScores.csv" file with latent trait scores of the focal trait and corresponding standard errors; 2) a "ScaleReliability.csv" file with the scale marginal reliability, which is defined as $\left(\sigma_{\theta}^{2}-\bar{\sigma}_{e}^{2}\right) / \sigma_{\theta}^{2}$, where $\sigma_{\theta}^{2}$ is the population variance of the trait scores, and $\bar{\sigma}_{e}^{2}$ is the average of the squared standard errors for trait scores; 3) the probabilities for plotting the IRF for each pair, and the IRF can be drew directly using the "surface" function in Microsoft Excel. An example IRF can be found in Figure 2.

\section{Discussion and Conclusion}

The MUPPscore script presented in this manual represents the first publicly available tool for estimating latent trait scores for the MUPP model for FC items. The script may help researchers get a better understanding of the MUPP format of testing and increase inquiries into and applications of IRT-based FC measurement in psychological research and practice. 


\section{References}

Albert, J. (2018). LearnBayes: Functions for Learning Bayesian Inference. R package version 2.15.1.

Boyce, A. S., Conway, J.S., Caputo, P.M. (2015). Development and Validation of Aon Hewitt's Personality Model and Adaptive Employee Personality Test (ADEPT-15). New York, NY: Aon Hewitt.

Chernyshenko, O. S., Stark, S., Prewett, M. S., Gray, A. a., Stilson, F. R., \& Tuttle, M. D. (2009). Normative scoring of multidimensional pairwise preference personality scales using IRT: empirical comparisons with other formats. Human Performance, 22(2), 105127.

Drasgow, F., Stark, S., Chernyshenko, O. S., Nye, C. D., Hulin, C. L., \& White, L. A. (2012). Development of the Tailored Adaptive Personality Assessment System (TAPAS) to Support Army Selection and Classification Decisions, (August), 1-108.

Guan, L., Carter, N. T., \& Lautenschlager, G.J. (April, 2015). Personality, Faking and ATIC: Can Forced-Choice Format Untangle Their Relationship? Paper presented for the $30^{\text {th }}$ Annual Meeting of the Society for Industrial and Organizational Psychology: Philadelphia, PA.

Hontangas, P. M., de la Torre, J., Ponsoda, V., Leenen, I., Morillo, D., \& Abad, F. J. (2015). Comparing Traditional and IRT Scoring of Forced-Choice Tests. Applied Psychological Measurement. http://doi.org/10.1177/0146621615585851

R Core Team (2018). R: A language and environment for statistical computing. Vienna, Austria: $R$ Foundation for Statistical Computing. Retrieved from http://www.R-project.org.

Roberts, J. S., Donoghue, J. R., \& Laughlin, J. E. (2000). A general item response theory model for unfolding unidimensional polytomous responses. Applied Psychological Measurement, 24, 3-32.

Stark, S., Chernyshenko, O. S., \& Drasgow, F. (2005). An IRT approach to constructing and scoring pairwise preference items involving stimuli on different dimensions: The multiunidimensional pairwise-preference model. Applied Psychological Measurement, 29(3), 184-203. 
Figure 1. Format of the Item Parameter File.

\begin{tabular}{|c|c|c|c|c|}
\hline 2 & A & B & $\mathrm{C}$ & \\
\hline 1 & Alpha & Delta & Tau & \\
\hline 2 & 0.91989 & 1.87539 & -2.419 & Statement 1: Focal trait \\
\hline 3 & 0.1737 & 10.6528 & -6.7758 & $\leftarrow$ Statement 2: Other trait \\
\hline 4 & 2.08324 & 0.82328 & -1.2661 & $\longleftarrow$ Statement 1: Focal trait \\
\hline 5 & 1.19928 & 4.01641 & -3.5203 & $\leftarrow$ Statement 2: Other trait \\
\hline 6 & 2.39414 & 0.65158 & -1.1774 & \\
\hline 7 & 1.38169 & 3.71992 & -3.2214 & \\
\hline 8 & 0.60325 & 3.38045 & -4.1987 & \\
\hline 9 & 0.72267 & 3.57175 & -2.6011 & \\
\hline 10 & 0.82505 & 2.46168 & -1.7647 & \\
\hline 11 & 0.66006 & 3.53449 & -4.0765 & \\
\hline 12 & 1.59665 & -0.9489 & -0.5186 & \\
\hline 13 & 0.42509 & -2.2652 & -3.0782 & \\
\hline 14 & 1.01428 & -1.6138 & -0.2814 & \\
\hline 15 & 0.99213 & -1.9585 & -3.0691 & \\
\hline 16 & 0.58305 & -1.461 & -2.5772 & \\
\hline 17 & 1.28519 & -0.9702 & -0.2375 & \\
\hline 18 & 1.60636 & -0.7872 & -0.7135 & \\
\hline 19 & 0.95978 & -0.445 & -0.8798 & \\
\hline 20 & 1.57116 & -0.6931 & -1.1329 & \\
\hline 21 & 0.78017 & -4.2625 & -3.6568 & \\
\hline
\end{tabular}


Figure 2. Example Item Response Function Using Data Generated by MUPPscore Using the Microsoft Excel Surface Charts function.

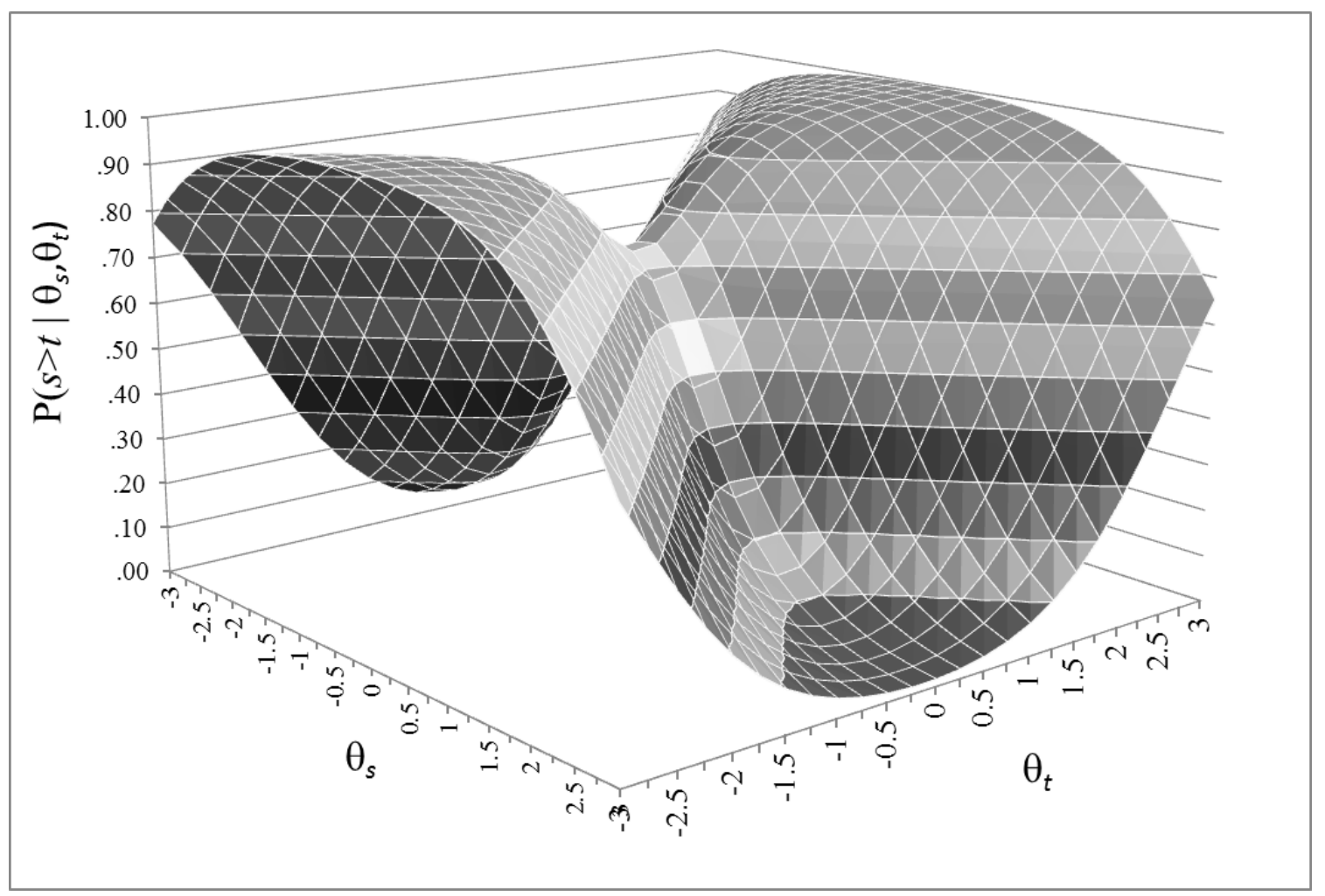

Note. Item parameters for $\theta_{s}$ were: $\alpha_{\mathrm{s}}=1.57, \delta_{\mathrm{s}}=-.35, \tau_{\mathrm{s}}=-1.03$; item parameters for $\theta_{t}$ were: $\alpha_{t}$ $=1.55, \delta_{t}=-.16, \tau_{t}=-.40$ 
Appendix A. R code for running the MUPPscore script.

setwd(" \newMUPPscore") \#\# Change working directory (Note: replace \with / or $\backslash \backslash$ )

if(!require(LearnBayes)) \{

\}.

install.packages ("LearnBayes")

library (LearnBayes)

$\mathrm{N}=1130$ \#\# Change sample size

PAIRS=10 \#\#Change number of pairs

$\mathrm{P}=29$ \#\# number of quadrature points (ONLY advanced users are allowed to change)

$\mathrm{PS}=841$ \#\# square the P (ONLY advanced users are allowed to change)

Parameters=read.csv ( "Parameters. csv", header $=\mathrm{T})$

Response=read.csv ("Responses.csv", header $=T$ )

NewResponse=Response[rep(1:nrow(Response), each=PS), ]

prior $=t(t(\operatorname{seq}(-3.5,3.5, .25)))$

PriorSForOne $=t(t(\operatorname{prior}[\operatorname{rep}(1: \operatorname{nrow}($ prior $)$, each=P $)])$,

PriorS=PriorSForOne $[\operatorname{rep}(1: P S, N)]$

PriorT=prior $[\operatorname{rep}(1: \mathrm{P}, \mathrm{N} * \mathrm{P})$,

PriorTForOne=prior $[\operatorname{rep}(1: P, P)$, ]

$\mathrm{mu}=\mathrm{c}(\theta, \theta)$

Sigma=matrix $(c(1,0,0,1), 2,2)$

PriorST=matrix (c (PriorS, PriorT) , , 2)

ProbS=dnorm (Priors, mu, Sigma, log=FALSE)

ProbT=dnorm (PriorT, mu, Sigma, log=FALSE)

ProbST $=$ dmnorm (PriorST, mu, Sigma, log=FALSE)

OneItemResponse $=$ matrix $($, nrow $=(P S * N), n c o l=1)$

PickedProb=matrix (, nrow $=(P S * N), n c o l=P A I R S)$

ThetaNumerator $=\operatorname{matrix}($, nrow $=\mathrm{N}, \mathrm{ncol}=1)$

ThetaDenominator $=$ matrix $($, nrow $=\mathrm{N}, \mathrm{ncol}=1)$

Theta=matrix $($, nrow $=\mathrm{N}, \mathrm{ncol}=1)$

StdErrorNumeratorAll=matrix $\left(\right.$, nrow $\left.=\left(P S^{*} N\right), n c o l=1\right)$

StdErrorNumerator $=$ matrix $($, nrow $=\mathrm{N}, \mathrm{ncol}=1)$

StdError=matrix ( , nrow $=\mathrm{N}, \mathrm{ncol}=1)$

ProbSgreaterTIRF=matrix (, nrow=PS, ncol=1)

for ( $i$ in 1 :PAIRS)

AlphaS=Parameters $[(2 * i-1), 1]$

BetaS=Parameter $[(2 * i-1), 2]$ 
TauS=Parameters $[(2 * i-1), 3]$

AlphaT=Parameters $[(2 * i), 1]$

BetaT=Parameters $[(2 * i), 2]$

TauT=Parameters $[(2 * i), 3]$

GammaS=1+exp (AlphaS*(3*(PriorS-BetaS)))+exp(AlphaS*( (PriorS-BetaS) -TauS)) +exp(AlphaS*(2*(PriorS-BetaS) -TauS)) GammaT=1+exp (AlphaT*(3*(PriorT-BetaT)) )+exp(AlphaT*((PriorT-BetaT) - TauT $))+\exp ($ AlphaT*(2*(PriorT-BetaT) $)$ TauT $))$

PS1 $=(\exp ($ AlphaS* ( PriorS-BetaS $)-$ TauS $))+\exp ($ AlphaS* $2 *($ PriorS-BetaS $)-T a u S))) /$ GammaS

PSO $=\left(1+\exp \left(\right.\right.$ AlphaS* $\left(3^{*}(\right.$ Priors-BetaS $\left.\left.\left.)\right)\right)\right) /$ GammaS

PT1 $=(\exp ($ AlphaT* $((\operatorname{PriorT}-B e t a T)-T a u T))+\exp (\operatorname{AlphaT*}(2 *(\operatorname{PriorT}-$ BetaT $)-$ TauT $))) /$ GammaT

PTO $=\left(1+\exp \left(\right.\right.$ AlphaT* $\left(3^{*}(\right.$ PriorT-BetaT $\left.\left.\left.)\right)\right)\right) /$ GammaT

ProbsgreaterT $=($ PS1*PTO) $/($ PS1*PT0+PSO*PT1)

ProbSlessT $=($ PSO*PT1) $/($ PS1*PT0+PS0*PT1)

GammaSIRF=1+exp (AlphaS*(3*(PriorSForOne-BetaS)))+exp(AlphaS*((PriorSForOne-BetaS) -TauS))+exp(AlphaS*(2*(PriorSForOne-BetaS) -TauS)) GammaTIRF=1+exp (AlphaT* (3*(PriorTForOne-BetaT) $))+\exp ($ AlphaT*( (PriorTForOne-BetaT)-TauT $))+\exp ($ AlphaT*(2*(PriorTForOne-BetaT) - TauT $))$ PS1IRF $=(\exp ($ AlphaS* $($ PriorSForOne-BetaS $)-$ TauS $))+\exp ($ AlphaS* $(2 *($ PriorSForOne-BetaS $)-$ TauS $))) /$ GammaSIRF PSOIRF $=(1+\exp ($ AlphaS* $(3 *($ PriorSForOne-BetaS $)))) /$ GammaSIRF

PT1IRF $=(\exp ($ AlphaT* $(($ PriorTForOne-BetaT $)-T a u T))+\exp ($ AlphaT* $(2 *(P r i o r T F o r O n e-B e t a T)-T a u T))) /$ GammaTIRF

PTOIRF $=(1+\exp ($ AlphaT* $(3 *($ PriorTForOne-BetaT $))) /$ GammaTIRF

ProbsgreaterTIRF=(PS1IRF*PTOIRF)/(PS1IRF*PTOIRF+PSOIRF*PT1IRF)

Table=matrix $($ ProbSgreaterTIRF, ncol=P)

colnames $($ Table $)=c($ seq $(-3.5,3.5, .25))$

rownames $($ Table $)=c(\operatorname{seq}(-3.5,3.5, .25))$

IRF Table=paste("IRFTable ",i, ".csv", sep=" ")

write.csv(Table, file=IRF_Table)

OneItemResponse $=$ NewResponse $[, i]$

for $(j$ in $1:(P S * N))$

\{

if (OneItemResponse $[j]==1)\{$

PickedProb $[j, i]=$ ProbSgreater $[j]\}$

if (OneItemResponse $[j]==0)\{$

PickedProb $[j, i]=$ ProbslessT $[j]\}$

else if (OneItemResponse $[j]==9$ )

PickedProb $[j, i]=1\}$

\}

rowProds=function $(X)\{\operatorname{apply}(\mathrm{X}, 1, \mathrm{FUN}=$ "prod" $)\}$

LVfA=rowProds (PickedProb)

LVf $=$ LVfA*ProbST

Af $=$ Probs $T$

NumeratorRaw $=t(t($ PriorS*LVf*Af $))$

DenominatorRaw $=t(t(L V f * A f))$ 


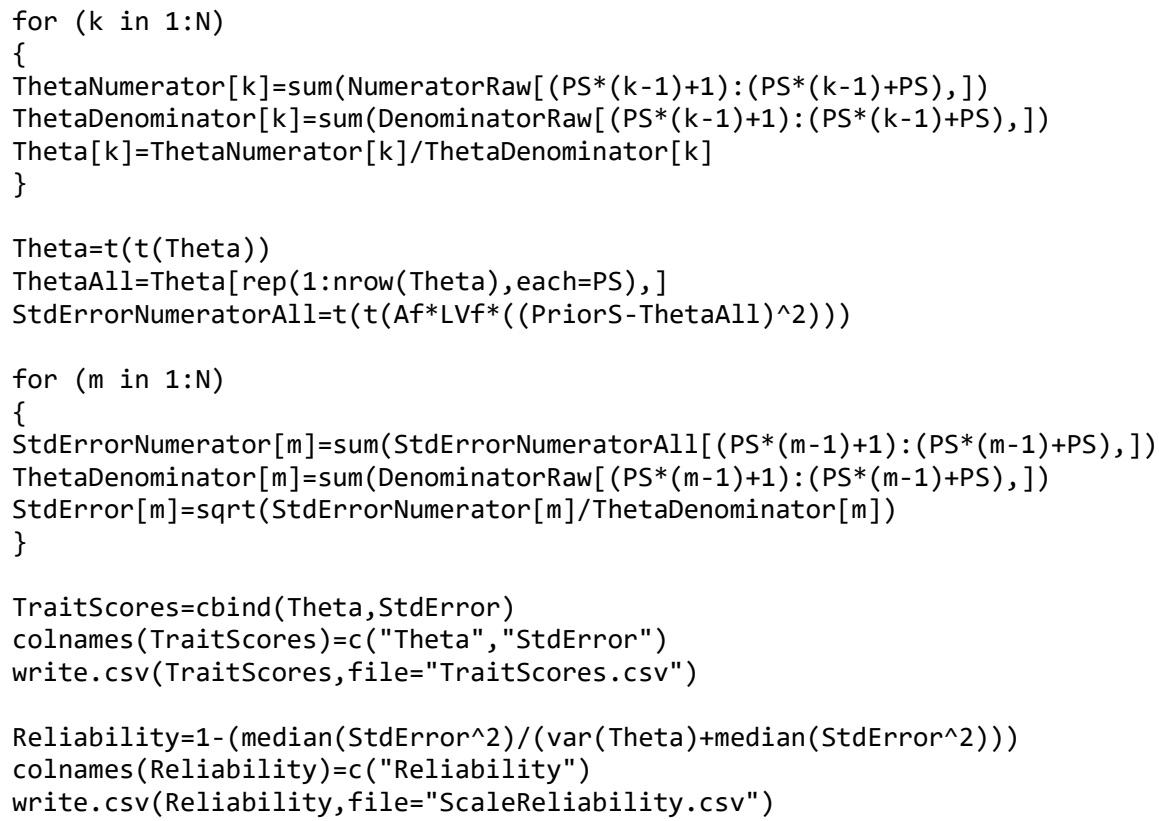

